\title{
AN EQUILIBRIUM MODEL FOR AN OTC DERIVATIVE MARKET UNDER A COUNTERPARTY RISK CONSTRAINT
}

\author{
KAZUHIRO TAKINO \\ Graduate School of Management \\ Nagoya University of Commerce and Business, Japan \\ takino@nucba.ac.jp
}

Received 26 February 2018

Accepted 2 October 2018

Published 12 November 2018

\begin{abstract}
In this study, we develop an equilibrium pricing model for an option contract with a counterparty risk, a collateral agreement, a counterparty risk constraint, and a threshold. Since we consider the option market to be an example of the derivatives market, we suppose that the buyer of an option has only countertparty risk of a seller defaulting. In addition, we consider a model where the buyer is allowed to enter into an option contract within an allocated amount of risk capital for counterparty risk, and requires (cash) collateral to the seller if the exposure exceeds the threshold. The counterparty risk is measured as a credit valuation adjustment. We provide an equilibrium pricing rule and an equilibrium volume formula by solving participants' static utility-maximization problems. Based on numerical simulations, we verify the mechanisms through which collateralization, risk capital, and the threshold affect the size of the overthe-counter (OTC) option market. Finally, we analyze the influence of the buyer's risk-aversion on the market, without collateralization. The results imply that the risk constraint might be a proxy for an investor's attitude towards risk.
\end{abstract}

Keywords: Equilibrium pricing; counterparty risk; counterparty risk constraint; collateralization; threshold; risk appetite.

JEL Classification: G10, G12, G13

\section{Introduction}

In this study, we consider the equilibrium pricing of an over-the-counter (OTC) derivative contract with counterparty risk, collateralization, and a counterparty risk constraint. Our study aims to verify the effect of collateralization on the OTC derivative contract under the restriction of counterparty risk. This restriction means that a market participant with a positive exposure to counterparty risk is able to

This is an Open Access article published by World Scientific Publishing Company. It is distributed under the terms of the Creative Commons Attribution 4.0 (CC-BY) License. Further distribution of this work is permitted, provided the original work is properly cited. 


\section{K. Takino}

have a derivative contract within an allocated amount of risk capital. In mathematical terms, the agent can have derivative contract position where the amount of counterparty risk is equal to or less than the amount of risk capital. In fact, the strengthening capital requirements for counterparty risks was incorporated into Basel III, launched in 2010 (BCBS 2010). That is, banks must now manage their counterparty risk exposures themselves. We call the restriction on the counterparty risk the 'counterparty risk constraint' or 'risk constraint' simply. In addition, we refer 'risk constraint is bound' or that we 'exhaust the risk capital' when the amount of the risk is equal to the amount of risk capital. Except for the counterparty risk constraint, Takino (2016b) has already studied the impact of collateralization on OTC derivative contracts (i.e. option and forward contracts). We construct an equilibrium pricing model by adding the counterparty risk constraint to the model of Takino (2016b), and describe how collateral agreement influences the OTC derivative contract under this constraint.

After the financial crisis in 2008, counterparty risks in OTC derivative contracts generated much attention from practitioners and researchers. Counterparty risk is known as the possibility of failing to meet the full payoff of a derivative contract. In order to hedge or eliminate counterparty risk, in 2013, the G20 decided that collateral agreements would be imposed on market participants in the OTC derivatives market. Derivative pricing models that include collateralization have recently been provided in several works (Fujii \& Takahashi 2013, Johannes \& Sundaresan 2003). These models provide pricing formulae via the risk-neutral pricing method, and show that collateralization is able to increase derivative prices.

In addition to derivatives pricing, previous studies have considered the effects of collateralization or margin on financial markets (Acharya \& Bisin 2014, Brunnermeier \& Pedersen 2009, Geanakoplos 1996, Takino 2016b). Collateral is delivered from the participant with negative exposure to the participant with positive exposure at fixed time(s) before the derivative contract matures. The payment to the participant with positive exposure is secured by the collateral if default occurs. On the one hand, when the participant with negative exposure defaults, she/he cannot recover the posted collateral. Therefore, collateralization affects investors' wealth and might influence participants' behaviors in the financial market. As shown in previous studies, the equilibrium pricing approach enables us to incorporate investors' behaviors into an asset price. The equilibrium pricing is also able to provide an equilibrium traded volume for a financial contract. Takino (2016b) showed that the impact on the option contract is more significant than that of the forward contract. While Takino (2016b) demonstrated that collateralization decreases the option volume overall (i.e. the trade-off relation between risk and liquidity), he also showed that an increase in collateral increases the volume of option contracts for small collateral amounts.

Collateral is used to hedge or eliminate the counterparty risk and is incurred by the participant with negative exposure if the amount of exposure exceeds a certain monetary level (called the threshold). On the other hand, the agent with positive 
exposure manages the counterparty risk on his/her own. The investors under the counterparty risk constraint are allocated risk capital for the counterparty risk and allowed to have positions or to trade derivatives within this amount. Danielsson et al. (2004) modeled the behaviors of the investors who posed a value-at-risk (VaR) constraint, and showed that the VaR constraint affects the asset return (including price and liquidity). Shin (2010) also lectures the effects of the VaR constraint on the asset market. Zhang \& Gao (2017) considered a dynamic portfolio selection problem under the VaR constraint, and demonstrated that the constraint reduces the invested proportion of risky assets in an optimal portfolio. For a general asset, Brunnermeier \& Pedersen (2009) considers how the margin influences market liquidity, showing that it is reduced by a decrease in capital.

In this study, we apply the counterparty risk constraint and the threshold, and construct an equilibrium pricing model for the derivative contract with a collateral agreement. In addition to Danielsson et al. (2004) and Zhang \& Gao (2017), Buss et al. (2016) considered a financial market model with financial regulations, such as investment constraints, borrowing constraints and so on. However, this is the first study to consider a constraint for the counterparty risk, to best of our knowledge. An equilibrium pricing formula for the option is provided, and we derive an equilibrium formula for the volume. We evaluate the marked-to-market (MtM) value of the derivative and the amount of counterparty risk using a pricing kernel (often called a stochastic discount factor), with an exponential utility to maintain a consistency with our equilibrium pricing approach. The amount of counterparty risk is evaluated as the credit value adjustment (CVA) in this study. Then, we observe the influences of collateralization, risk capital (or the risk constraint), and the threshold on the equilibrium price and volume of the option contract. In particular, we find that the pricing formulae depend on the amount of risk capital when the participant exhausts the risk capital. To highlight the effects of the risk constraint on the derivative contract, we also provide equilibrium formulae without collateralization, and investigate the effect of an investor's risk-aversion on the derivative contract.

The results in this study rely on numerical implementations. We show that the equilibrium price and volume of an option increase when the amount of collateral increases. This characteristic has already been demonstrated by Takino (2016b) for a particular parameter set. Our study provides an explicit mechanism to show this property through the counterparty risk constraint. The increase in collateral decreases the amount of the counterparty risk. This relaxes the constraint of the counterparty risk for the option buyer and increases demand for the claim. In fact, we demonstrate that the price and volume when the risk constraint is not bound are larger than those when the constraint is bound. Conversely, tightening the risk constraint decreases the price and volume. That is, we show that collateralization affects the derivative contract under counterparty risk through the counterparty risk constraint.

We obtained the following with regard to the allocated risk capital and the threshold affect on the counterparty risk constraint, because these change the 


\section{K. Takino}

collateral amount to be posted. We show that an increase in the risk capital or a decrease in the threshold increases the equilibrium price and volume (i.e. market size) of the claim. Conversely, to decrease the market size of the OTC derivative market, we can reduce the risk capital for counterparty risk or raise the threshold. Hence, our findings show that risk capital and the threshold might be devices that can be used to control the market like other financial regulations as demonstrated by Buss et al. (2016).

Finally, we investigate the effect of a buyer's risk aversion on the derivative contract without collateralization. The numerical result demonstrates that the price and volume decrease when an investor becomes more risk averse. This is equivalent to the influence of a decrease in risk capital or an increase in the threshold on the derivative contract. That is, the result indicates that the risk constraint might be a proxy for an investor's attitude to risk, and is able to adjust the market without controlling an investor's risk aversion. Risk capital is money that allows investors to make a loss from asset trading. Thus, the allocated risk capital indicates a risk appetite. Some previous studies have found that the concept of risk appetite does not only include risk aversion (Danielsson et al. 2010, Illing \& Aaron 2005), because it might change according to economic or management conditions, as mentioned by Danielsson et al. (2010). However, Danielsson et al. (2010) considers the behavior of a risk neutral investor with risk capital restricted with $\mathrm{VaR}$, and shows that the risk capital makes the investor to behave as a risk averse investor. Our result also highlights a consistency between risk aversion and risk appetite.

The remainder of the paper is organized as follows. In the next section, we introduce a financial market model with a collateral agreement and a counterparty risk constraint. We also provide a pricing kernel, which we use to evaluate the amount of the counterparty risk and collateral from the utility-maximization problems for market participants with an exponential utility. In Sec. 3, we derive the equilibrium price and volume for the option contract under the counterparty risk constraint in the context of the exponential utility framework. In Sec. 4, we observe the effects of collateralization on price and volume for the claim using a numerical method. In the numerical implementation, we use a continuous time model and a Monte Carlo simulation. We conclude the paper in Sec. 5.

\section{Model and Collateralization}

In this section, we set a financial market model for an OTC derivative market with a counterparty risk. The market model is constructed based on Takino (2016b).

\subsection{Financial markets}

We first set a probability space $(\Omega, \mathcal{F}, P)$ and denote the element of $\Omega$ by $\omega$.

There are two market participants in our financial market. They respectively invest in a risk-free asset (typically a bank account) and two risky assets (typically stocks). 
We further assume that the risky asset invested in by an agent differs from that held by another investor, for all participants, and that the amount invested in a risky asset is given exogenously. The risk-free asset value at time $t(0 \leq t \leq T)$ is denoted by $B_{t}=e^{r t}$ with a constant risk-free rate $r$, and the risky asset value at time $t$ is expressed by $S_{t}^{j}$, held by participant $j$, where $T$ denotes the maturity date of the derivative. We denote $\pi_{j}$ as the amount invested in the risky asset by agent $j(j=l, s)$.

In addition to the risky assets, we suppose there is a European-type derivative written on a risky asset $Y$, with a payoff function at maturity $T$ given by

$$
H(T, \omega):=H\left(T, Y_{T}(\omega)\right)
$$

and the price of the underlying asset at time $t$ denoted by $Y_{t}(0 \leq t \leq T)$. We also assume that the price of the underlying asset is correlated with the risky assets. We do not restrict the underlying asset $Y$ in terms of whether it is traded in our economy. Thus, the underlying asset includes a stock index, a commodity price, an average temperature, and so on. We denote the long-holder of the derivative by ' $l$ ' and the short-holder by ' $s$ '. Furthermore, they behave as price takers in the financial markets included the derivative market.

We assume that there is a counterparty risk in the derivative contract. The counterparty risk is the possibility that a participant will fail to meet the full payout of $H(T, \omega)$. In order to reduce or eliminate the loss due to the counterparty risk, the agent with positive exposure can require (cash) collateral from the counterparty with negative exposure at the MtM date. Takino (2016a) shows that the affect of collateralization on an option contract is more significant than it is on a swap contract. Therefore, in our study, we only consider an option contract as an example of a derivative contracts. Hence, the long-holder (buyer) of the option only has positive exposure for the derivative contract; that is, the counterparty risk is unilateral. We represent the event of default by an indicator function $1_{D}(\omega)$, and denote the recovery rate of a default payment by $\eta(T, \omega)$. We also suppose that both are determined from the short-holder's risky asset value $S_{T}^{s}$ at maturity. Under these settings, the option payoff $\hat{H}$ with counterparty risk is represented by

$$
\begin{aligned}
\hat{H}(T, \omega) & =H(T, \omega)\left(1-1_{D}(\omega)\right)+\eta(T, \omega) H(T, \omega) 1_{D}(\omega) \\
& =H(T, \omega)-H(T, \omega)(1-\eta(T, \omega)) 1_{D}(\omega) .
\end{aligned}
$$

From (2.1), we observe that the payoff function with counterparty risk is decomposed into a payoff without counterparty risk and a loss due to counterparty risk.

\subsection{Threshold and net exposure}

We introduce a threshold for a derivatives contract. The threshold is a sort of credit line, and collateral is called from the participant with positive exposure to the derivatives contract if the exposure exceeds the threshold (Gregory 2010). Denoting 


\section{K. Takino}

the threshold and the exposure at time $t$ by $M$ and $\tilde{V}_{t}$, respectively, the participant with negative exposure is required to provide collateral at contract date $t=0$ if

$$
\tilde{V}_{0}>M \text {. }
$$

We then define the net exposure $V_{0}$ at time 0 as

$$
V_{0}=\max \left(\tilde{V}_{0}-M, 0\right) \text {. }
$$

We use an MtM value as an exposure amount and the MtM is only executed at the contract date of the derivatives contract (i.e. $t=0$ ). We suppose that $\tilde{V}_{t}$ is calculated with a pricing kernel or stochastic discount factor to maintain a consistency with our equilibrium pricing approach. Thus, the MtM value of the option at time $t$ is

$$
\tilde{V}_{t}=E\left[\frac{\mathcal{E}(T)}{\mathcal{E}(t)} H(T)\right],
$$

where $\mathcal{E}$ is the pricing kernel determined from $\mathcal{E}(0)=1$. This pricing formula is known as the economic premium principle. The economic premium principle approach was initiated by Bühlmann (1980). Since then, several researchers have applied the formula to price contingent claims (Iwaki et al. 2001, Iwaki 2002, Kijima et al. 2010, Takino 2016a).

We note that the threshold is not used to evaluate the counterparty risk (or credit risk) and is only used to determine the collateral amount. The counterparty risk is calculated using a CVA, as introduced below.

\subsection{Collateral agreement}

Cash collateral is posted to the agent with positive exposure from the counterparty with negative exposure if the exposure exceeds the threshold. We assume that the collateral is deposited into a risk-free asset to eliminate a collateral default. ${ }^{\text {a }}$ The collateral amount is determined from the net exposure $V$ and coverage ratio $\phi(\geq 0)$. The coverage ratio determines how much of the risk the investor wants to eliminate, and the actual settled amount of collateral is determined by $\phi$ in this study. If the agent wants to fully cover the potential loss from a default, then she/he sets $\phi$ more than $100 \%$. The case of $\phi=0 \%$ corresponds to the case of no collateral. Then, the called collateral amount at the contract date is

$$
\phi V_{0} .
$$

The option buyer loses amount $(1-\eta(T, \omega)) H(T, \omega)$ when the counterparty defaults. The collateral is aimed to cover this loss. Of course, there is a possibility that the collateral amount is larger than the default loss; that is,

$$
\phi B_{T} V_{0}>(1-\eta(T, \omega)) H(T, \omega) .
$$

a We call the possibility of failing to return the collateral 'collateral default'. 
In this case, we suppose that the residual cash amount $\phi B_{T} V_{0}-(1-\eta) H(T, \omega)$ is returned to the seller who posted the collateral. Therefore, the collateral amount $C(T, \omega ; \phi)$ that the buyer can obtain at the seller's default with coverage ratio $\phi$, is $C(T, \omega ; \phi)$

$$
\begin{aligned}
& =\phi B_{T} V_{0} 1_{D}(\omega)-\left\{\begin{array}{cl}
\left(\phi B_{T} V_{0}-(1-\eta(T, \omega))\right. & \\
\times H(T, \omega)) 1_{D}(\omega), & \text { if } \phi B_{T} V_{0}>(1-\eta(T, \omega)) H(T, \omega) \\
0, & \text { if } \phi B_{T} V_{0} \leq(1-\eta(T, \omega)) H(T, \omega)
\end{array}\right. \\
& =\min \left(\phi B_{T} V_{0},(1-\eta(T, \omega)) H(T, \omega)\right) 1_{D}(\omega) \text {. }
\end{aligned}
$$

Note that, although we can omit the default indicator function $1_{D}(\omega)$ in $(2.3)$, we incorporate it in $C(T, \omega ; \phi)$ for the following discussion.

Finally, we provide the payoff function including a collateral agreement. The buyer of the claim receives full payment $H$ if the seller does not default, and the longholder obtains the default payment $\eta \times H$ and payment $C$ from the collateral agreement. Therefore, the payoff function with a collateral agreement is given by

$$
\begin{aligned}
g(T, \omega) & =H(T, \omega)\left(1-1_{D}(\omega)\right)+\eta(T, \omega) H(T, \omega) 1_{D}(\omega)+C(T, \omega ; \phi) \\
& =\hat{H}(T, \omega)+C(T, \omega ; \phi) .
\end{aligned}
$$

The last equality in (2.4) is from (2.1) and (2.3). By the economic premium principle, the price $p$ of claim $g$ is given by

$$
p=E[\mathcal{E}(T) g(T)] .
$$

The pricing formula (2.5) shows that the defaultable claim $\hat{H}$ and the collateral value $C$ are given by the pricing kernel. Note that pricing formula (2.5) does not reflect the counterparty risk constraint. For the pricing formula under the counterparty risk constraint, we also provide an equilibrium pricing rule to incorporate the constraint.

\subsection{Counterparty risk constraint}

In this section, we introduce a counterparty risk constraint. That is, the investor with this constraint should trade derivatives such that the amount of counterparty risk is less than or equal to the allocated risk capital. In this work, the buyer of the option only exposed the counterparty risk. The buyer, hence, behaves under such a constraint.

We suppose that the counterparty risk is evaluated by CVA criterion. This criterion has recently become popular among financial institutions, where the risk management division requires the amount of the CVA from traders who enter derivative contracts with positive exposure in order to hedge against counterparty risks. For more detail, we recommend Brigo \& Masetti (2005), Fujii \& Takahashi (2013) or Gregory (2010).

While the CVA increases with an increase in the counterparty risk, it is reduced by collateralization. Thus, the CVA is defined as the difference between payoffs with 


\section{K. Takino}

or without the counterparty risk minus the collateral amount. That is, the CVA with the collateral for $k$-claims is

$$
\begin{aligned}
\operatorname{CVA}(k, \phi) & =E[\mathcal{E}(T) k H(T, \omega)]-E[\mathcal{E}(T) k \hat{H}(T, \omega)]-E[\mathcal{E}(T) k C(T, \omega ; \phi)] \\
& =k \max \left(E\left[\mathcal{E}(T)\left\{(1-\eta(T, \omega)) H(T, \omega)-\phi B_{T} V_{0}\right\} 1_{D}(\omega)\right], 0\right) \\
& =k \operatorname{CVA}(\phi),
\end{aligned}
$$

where $\operatorname{CVA}(\phi)=\max \left(E\left[\mathcal{E}(T)\left\{(1-\eta(T, \omega)) H(T, \omega)-\phi B_{T} V_{0}\right\} 1_{D}(\omega)\right], 0\right)$.

We exogenously provide the allocated risk capital for the counterparty risk $L(>0)$. The buyer of the option then has to order the derivative contract such that

$$
\operatorname{CVA}(k, \phi)=k \operatorname{CVA}(\phi) \leq L .
$$

From this inequality, the risk constraint is relaxed with an increase in $L$. Now, we suppose that threshold $M$ is increased. The increase in $M$ reduces the net exposure $V_{0}$, and this decreases the required collateral amount, and simultaneously increases $\operatorname{CVA}(\phi)$, since

$$
\max (x, 0) \geq \max (y, 0),
$$

for any $x \geq y$. Thus, when the threshold $M$ increases, the amount of the counterparty risk reaches $L$ and the counterparty risk constraint is bound.

\subsection{Participants' preferences and wealth}

In order to derive the demand and supply functions, we consider utility-maximization problems for total wealth, consisting of a bank account, the risky asset, and the derivative. We denote $W_{t}^{j}$ as the wealth of participant $j(j=l, s)$ at time $t$. In addition, the amount invested in the risky asset by participant $j$ is denoted by $\pi_{j}$, and the volume of the claim participant $j$ is willing to trade is denoted by $k_{j}$. We assume that $k_{j}>0$ for each $j \in\{l, s\}$.

\subsubsection{Participants' preferences}

We suppose that participants' preferences are measured by the utility function $U^{j}(j=l, s)$, and that participants have an exponential utility, that is, the utility function of participant $j$ is represented by

$$
U^{j}(x)=-\frac{1}{\gamma_{j}}\left(1-e^{-\gamma_{j} x}\right),
$$

for $x \geq 0$, where $\gamma_{j}$ is the risk-aversion coefficient of participant $j$.

\subsubsection{Buyer's wealth equation}

The buyer with initial wealth $X_{0}^{l}=x_{l}(>0)$ invests money in the risky asset and the derivative contract. The buyer might have an incentive to purchase an option to hedge the risk of the risky asset. The rest of the money is deposited in the risk-free asset. Note that the buyer has positive exposure for the derivative contract and 
she/he must receive the collateral $\phi V_{0}$ per claim. Then, the buyer's amount $w_{l}$ invested in the risk-free asset is

$$
w_{l}=x_{l}-\frac{\pi_{l}}{S_{0}^{l}} S_{0}^{l}-k_{l} p+k_{l} \phi V_{0}=x_{l}-\pi_{l}-k_{l}\left(p-\phi V_{0}\right) .
$$

Next, at maturity, the buyer should return the posted collateral $\phi B_{T} V_{0}$ to the seller if she/he does not default. On the one hand, the buyer pays even if the seller defaults, whenever the collateral amount is larger than the default loss; that is, $\phi B_{T} V_{0}>(1-\eta) H(T)$. Therefore, the terminal wealth for the buyer is

$$
\begin{aligned}
X_{T}^{l}(\omega)= & w_{l} B_{T}+\frac{\pi_{l}}{S_{0}^{l}} S_{T}^{l}(\omega)+k_{l} \hat{H}(T, \omega)-k_{l} \phi B_{T} V_{0}\left(1-1_{D}(\omega)\right) \\
& - \begin{cases}k_{l}\left(\phi B_{T} V_{0}-(1-\eta) H(T, \omega)\right) 1_{D}(\omega), & \phi B_{T} V_{0}>(1-\eta(T, \omega)) H(T, \omega) \\
0, & \phi B_{T} V_{0} \leq(1-\eta(T, \omega)) H(T, \omega)\end{cases} \\
= & \left(x_{l}-\pi_{l}-k_{l} p\right) B_{T}+\frac{\pi_{l}}{S_{0}^{l}} S_{T}^{l}(\omega)+k_{l} \hat{H}(T, \omega) \\
& +k_{l} \min \left(\phi B_{T} V_{0},(1-\eta(T, \omega)) H(T, \omega)\right) 1_{D}(\omega) \\
= & \left(x_{l}-\pi_{l}-k_{l} p\right) B_{T}+\frac{\pi_{l}}{S_{0}^{l}} S_{T}^{l}(\omega)+k_{l}(\hat{H}(T, \omega)+C(T, \omega ; \phi)) \\
= & \left(x_{l}-\pi_{l}-k_{l} p\right) B_{T}+\frac{\pi_{l}}{S_{0}^{l}} S_{T}^{l}(\omega)+k_{l} g(T, \omega) .
\end{aligned}
$$

\subsubsection{Seller's wealth equation}

The seller with initial wealth $X_{0}^{s}=x_{s}(>0)$ invests money in the risky asset in the same way as the buyer and sells the option. On the other hand, the position of the claim and the delivery of collateral are opposite to the buyer's case. The terminal wealth of the seller, therefore, is

$$
X_{T}^{s}(\omega)=\left(x_{s}-\pi_{s}+k_{s} p\right) B_{T}+\frac{\pi_{s}}{S_{0}^{s}} S_{T}^{s}-k_{s} g(T, \omega)
$$

\subsection{Pricing kernel}

Lastly, we determine the pricing kernel $\mathcal{E}$. The pricing kernel is derived from the utility-maximization problem and the definition of the equilibrium.

We first set the utility-maximization problems. We suppose that market participant $j$ determines the contract volume $k_{j}$ of the claim to maximize the (expected) utility for her/his terminal wealth. That is, the maximization problem of participant $j$ is defined by

$$
\max _{k_{j}} E\left[U^{j}\left(X_{T}^{j}\right)\right]
$$

for $j=l, s$.

Next, we define the market equilibrium. 
Definition 2.1 (Market Equilibrium). The market is in equilibrium if

(1) $\sum_{j \in\{l, s\}}\left(x_{j}-\pi_{j}\right)=R_{0}$,

(2) $\sum_{j \in\{l, s\}} \frac{\pi_{j}}{S_{0}^{j}} S_{T}^{j}(\omega)=R_{T}(\omega)$,

(3) $\sum_{j \in\{l, s\}} \delta_{j} k_{j}=0$,

where $k_{j} \geq 0$ for all $j$.

From (2.7) and (2.8), we represent

$$
X_{T}^{j}(\omega)=\left(x_{j}-\pi_{j}-\delta_{j} k_{j} p\right) B_{T}+\frac{\pi_{j}}{S_{0}^{j}} S_{T}^{j}(\omega)+\delta_{j} k_{j} g(T, \omega),
$$

for $j=l, s$, where $p$ is given in (2.5). Solving utility-maximization problem (2.9) with respect to $k_{j}$ and using Definition 2.1 provides the pricing kernel $\mathcal{E}$. We omit the proof of theorem, which follows the proof of Theorem 3.1 in Takino (2016a).

Theorem 2.1. Under market equilibrium (Definition 2.1), the pricing kernel $\mathcal{E}$ is given by

$$
\mathcal{E}(T)=\frac{e^{-\gamma R_{T}(\omega)}}{B_{T} E\left[e^{\left.-\gamma R_{T}\right]}\right.},
$$

where $\frac{1}{\gamma}=\sum_{j=1}^{J} \frac{1}{\gamma_{j}}$.

Pricing kernel $\mathcal{E}$ is used to determine the value of the $\operatorname{MtM} \tilde{V}$ and $\operatorname{CVA}(k, \phi)$. Unfortunately, we cannot determine the equilibrium volume of the option contract. In order to derive the formula of the equilibrium volume explicitly, we derive the equilibrium from a linearly approximated exponential utility (2.6) in the following section. This also provides a pricing formula under the counterparty risk constraint.

\section{Demand/Supply Functions and Market Equilibrium}

In this section, we derive the equilibrium price and volume for the option with a collateral agreement under the counterparty risk constraint. The demand and supply functions are obtained by solving the investors' utility-maximization problem with respect to claim volume $k_{j}(j=l, s)$. Recall, participants have exponential utility (2.6). By taking the Taylor expansion of the expected utility for small $\gamma_{j}(j=l, s)$, we have the mean-variance form of the expected exponential utility,

$$
E\left[U^{j}\left(X_{T}^{j}\right)\right]=E\left[X_{T}^{j}\right]-\frac{\gamma_{j}}{2} \operatorname{Var}\left[X_{T}^{j}\right]
$$

where $\gamma_{j}$ is the risk aversion of participant $j$. Acharya \& Bisin (2014), Bessembinder \& Lemmon (2002), and Takino (2016b) used the mean-variance criterion as the expected utility of an investor to derive the equilibrium price and volume for assets. 


\subsection{Demand functions}

We first formulate the buyer's utility maximization problem under the counterparty risk constraint. The buyer's problem under the constraint is

$$
\begin{array}{ll}
\max _{k_{l}} & E\left[U^{l}\left(X_{T}^{l}\right)\right] \\
\text { s.t. } & k_{l} \operatorname{CVA}(\phi) \leq L .
\end{array}
$$

The expected utility of the buyer for terminal wealth (2.7) is given by

$$
E\left[U^{l}\left(X_{T}^{l}\right)\right]=E\left[X_{T}^{l}\right]-\frac{\gamma_{l}}{2} \operatorname{Var}\left[X_{T}^{l}\right]
$$

where

$$
\begin{gathered}
E\left[X_{T}^{l}\right]=\left(x_{l}-\pi_{l}-k_{l} p\right) B_{T}+\frac{\pi_{l}}{S_{0}^{l}} E\left[S_{T}^{l}\right]+k_{l} E[g(T)] \\
\operatorname{Var}\left[X_{T}^{l}\right]=\left(\frac{\pi_{l}}{S_{0}^{l}}\right)^{2} \operatorname{Var}\left[S_{T}^{l}\right]+k_{l}^{2} \operatorname{Var}[g(T)]+2 \frac{\pi_{l}}{S_{0}^{l}} k_{l} \operatorname{Cov}\left[S_{T}^{l}, g(T)\right] .
\end{gathered}
$$

We first define the Lagrange function $\mathcal{L}^{l}$ as

$$
\mathcal{L}^{l}=E\left[U^{l}\left(X_{T}^{l}\right)\right]+\lambda_{l}\left(L-k_{l} \operatorname{CVA}(\phi)\right) .
$$

The KKT condition for the maximization problem (3.2) is given by

$$
\begin{aligned}
\frac{\partial \mathcal{L}^{l}}{\partial k_{l}} & =\partial_{l} E\left[U^{l}\left(X_{T}^{l}\right)\right]-\lambda_{l} \operatorname{CVA}(\phi)=0, \\
\frac{\partial \mathcal{L}^{l}}{\partial \lambda_{l}} & =L-k_{l} \operatorname{CVA}(\phi) \geq 0, \\
\lambda_{l} \frac{\partial \mathcal{L}^{l}}{\partial \lambda_{l}} & =\lambda_{l}\left\{L-k_{l} \operatorname{CVA}(\phi)\right\}=0,
\end{aligned}
$$

where $\lambda_{l}(\geq 0)$ is a Lagrange multiplier and

$$
\begin{aligned}
\partial_{l} E\left[U^{l}\left(X_{T}^{l}\right)\right] & :=\frac{\partial E\left[U^{l}\left(X_{T}^{l}\right)\right]}{\partial k_{l}} \\
& =-p B_{T}+E[g(T)]-\gamma_{l} k_{l} \operatorname{Var}[g(T)]-\gamma_{l} \frac{\pi_{l}}{S_{0}^{l}} \operatorname{Cov}\left[S_{T}^{l}, g(T)\right] .
\end{aligned}
$$

The buyer's problem is solved as follows.

(1) $\lambda_{l}>0$ Case

When $\lambda_{l}>0$, from (3.5), we have

$$
L-k_{l} \operatorname{CVA}(\phi)=0 .
$$

By the assumption of $L>0$, (3.6) leads to $\operatorname{CVA}(\phi)>0$. From (3.6), $k_{l}$ is given by

$$
k_{l}=\frac{L}{\operatorname{CVA}(\phi)} \text {. }
$$




\section{K. Takino}

From (3.3), we have

$$
\lambda_{l}=\frac{\partial_{l} E\left[U^{l}\left(X_{T}^{l}\right)\right]}{\operatorname{CVA}(\phi)} .
$$

From $\operatorname{CVA}(\phi)>0$, if $\partial_{l} E\left[U^{l}\left(X_{T}^{l}\right)\right]>0$, then we have

$$
\lambda_{l}>0
$$

and the optimal $k_{l}$ is given by

$$
k_{l}^{*}=\frac{L}{\operatorname{CVA}(\phi)} .
$$

(2) $\lambda_{l}=0$ (or $\left.\partial_{l} E\left[\boldsymbol{U}^{l}\left(\boldsymbol{X}_{T}^{l}\right)\right] \leq \mathbf{0}\right)$ Case

If $\partial_{l} E\left[U^{l}\left(X_{T}^{l}\right)\right] \leq 0$, then, from (3.7) and the fact that $\lambda_{l} \geq 0$, we have $\lambda_{l}=0$. This time, by (3.3), the optimal $k_{l}$ is the solution of

$$
\partial_{l} E\left[U^{l}\left(X_{T}^{l}\right)\right]=0
$$

That is, the optimal $k_{l}$ is

$$
k_{l}^{*}=-\frac{1}{\gamma_{l} \operatorname{Var}[g(T)]}\left\{p B_{T}-E[g(T)]+\gamma_{l} \frac{\pi_{l}}{S_{0}^{l}} \operatorname{Cov}\left[S_{T}^{l}, g(T)\right]\right\} .
$$

\subsection{Supply function}

The supply function is obtained from the seller's utility-maximization problem for her/his terminal wealth. If the seller has negative exposure, then she/he is not confined by the counterparty risk constraint. ${ }^{\mathrm{b}}$ Therefore, the risk constraint for the seller is released and her/his maximization problem is given by

$$
\max _{k_{s}} E\left[U^{s}\left(X_{T}^{s}\right)\right]
$$

The expected utility of the seller for (2.8) is given by

$$
E\left[U^{s}\left(X_{T}^{s}\right)\right]=E\left[X_{T}^{s}\right]-\frac{\gamma_{s}}{2} \operatorname{Var}\left[X_{T}^{s}\right]
$$

where

$$
\begin{gathered}
E\left[X_{T}^{s}\right]=\left(x_{s}-\pi_{s}+k_{s} p\right) B_{T}+\frac{\pi_{s}}{S_{0}^{s}} E\left[S_{T}^{s}\right]-k_{s} E[g(T)] \\
\operatorname{Var}\left[X_{T}^{s}\right]=\left(\frac{\pi_{s}}{S_{0}^{s}}\right)^{2} \operatorname{Var}\left[S_{T}^{s}\right]+k_{s}^{2} \operatorname{Var}[g(T)]-2 \frac{\pi_{s}}{S_{0}^{s}} k_{s} \operatorname{Cov}\left[S_{T}^{s}, g(T)\right]
\end{gathered}
$$

\footnotetext{
${ }^{\mathrm{b}}$ In fact, the seller's CVA is nonpositive for the option contract. Thus, the constraint $k_{s} \operatorname{CVA}(\phi) \leq L$ is always satisfied even if the seller is imposed the counterparty risk constraint.
} 
From the first-order condition, we have

$$
p B_{T}-E[g(T)]-\gamma_{s} k_{s} \operatorname{Var}[g(T)]+\gamma_{s} \frac{\pi_{s}}{S_{0}^{s}} \operatorname{Cov}\left[S_{T}^{s}, g(T)\right]=0
$$

The supply function is thus

$$
k_{s}^{*}=\frac{1}{\gamma_{s} \operatorname{Var}[g(T)]}\left\{p B_{T}-E[g(T)]+\gamma_{s} \frac{\pi_{s}}{S_{0}^{s}} \operatorname{Cov}\left[S_{T}^{s}, g(T)\right]\right\} .
$$

\subsection{Equilibria}

We provide the equilibrium price and volume using the demand and supply functions derived earlier. The demand function used to derive the equilibria differs from the value of $\partial_{l} E\left[U^{l}\left(X_{T}^{l}\right)\right]$ or $\lambda_{l}$.

\section{(1) $\partial_{l} E\left[U^{l}\left(X_{T}^{l}\right)\right]>0\left(\lambda_{l}>0\right)$ Case}

The demand function is given by (3.8). From the equilibrium condition $k_{l}^{*}=k_{s}^{*}$, the equilibrium price $p^{*}$ is

$$
p^{*}=\frac{1}{B_{T}}\left\{E[g(T)]-\gamma_{s} \frac{\pi_{s}}{S_{0}^{s}} \operatorname{Cov}\left[S_{T}^{s}, g(T)\right]+\gamma_{s} \frac{L}{\operatorname{CVA}(\phi)} \operatorname{Var}[g(T)]\right\} .
$$

At this point, from (2.5), the derivative price used to estimate the collateral value is represented by

$$
\begin{aligned}
p & =E[\mathcal{E}(T) g(T)] \\
& =\frac{1}{B_{T}} E[g(T)]+\operatorname{Cov}[\mathcal{E}(T), g(T)] .
\end{aligned}
$$

Here, Eq. (3.11) shows that the derivative price is given by the expected discount payoff, adjusted by a covariance term of the pricing kernel, and the derivative payoff when we use the physical measure. The pricing kernel $\mathcal{E}$ of (2.10) includes the risk-aversions of the participants, that is, $\gamma_{b}$ and $\gamma_{s}$. Therefore, we consider that this covariance term with risk-aversions adjusts the risk instead of the change of measure. On the other hand, the second and third terms in (3.10) also play a role in adjusting the expected discount payoff under the physical measure. While $\gamma_{s}$ is explicitly included in the pricing formula (3.10), $\gamma_{b}$ does not appear in the formula. Alternatively, we can find the term of $L / \mathrm{CVA}(\phi)$ in (3.10). Here, $L$ and $\mathrm{CVA}(\cdot)$ are related to the risk constraint posed to the buyer. Thus, we consider that the equilibrium price is reflected in the buyer's attitude to risk through the risk constraint. In fact, we find that $L / \mathrm{CVA}(\phi)$ plays a role in the risk-aversion of the buyer in Sec. 4.3.

The equilibrium volume $k^{*}$ is

$$
k^{*}=\frac{L}{\operatorname{CVA}(\phi)} .
$$


Remark 3.1. When $\operatorname{CVA}(\phi)=0$, the price and volume are not finite. However, $\operatorname{CVA}(\phi)=0$ and $k_{l}^{2} \mathrm{CVA}^{2}(\phi)<L^{2}$ are equivalent because $L>0$. This leads to that $\lambda_{l}=0$ for the KKT condition, and then the case of $\partial_{l} E\left[U^{l}\left(X_{T}^{l}\right)\right]>0$ is rejected.

(2) $\partial_{l} \boldsymbol{E}\left[\boldsymbol{U}^{l}\left(\boldsymbol{X}_{T}^{l}\right)\right] \leq \mathbf{0}\left(\lambda_{l}=\mathbf{0}\right)$ Case

The demand function is given by (3.9). From the equilibrium condition $k_{l}=k_{s}$, the equilibrium price $p^{*}$ is

$$
p^{*}=\frac{1}{B_{T}} E[g(T)]-\frac{1}{B_{T}} \gamma \operatorname{Cov}\left[R_{T}, g(T)\right],
$$

where $R_{T}$ is defined in Definition 2.1. Substituting (3.13) into (3.9) yields the equilibrium volume $k^{*}$,

$$
k^{*}=\frac{\gamma_{s} \frac{\pi_{s}}{S_{0}^{s}} \operatorname{Cov}\left[S_{T}^{s}, g(T)\right]-\gamma_{l} \frac{\pi_{l}}{S_{0}^{l}} \operatorname{Cov}\left[S_{T}^{l}, g(T)\right]}{\left(\gamma_{l}+\gamma_{s}\right) \operatorname{Var}[g(T)]} .
$$

\subsubsection{Sensitivity analysis}

We can easily observe the effects of the counterparty risk constraint on the equilibria without relying on the numerical scheme in the case of $\partial_{l} E\left[U^{l}\left(X_{T}^{l}\right)\right]>0$. This case corresponds to $k_{l} \mathrm{CVA}(\phi)=L$; that is, the risk constraint is bound.

First, we consider the influence on the volume. The demand function and equilibrium volume are equal:

$$
k_{l}^{*}=k^{*}=\frac{L}{\operatorname{CVA}(\phi)} .
$$

Differentiating $k_{l}^{*}$ and $k^{*}$ with respect to $L$, we have

$$
\frac{\partial k_{l}^{*}}{\partial L}=\frac{\partial k^{*}}{\partial L}=\frac{1}{\operatorname{CVA}(\phi)}>0 .
$$

Thus, the risk capital $L$ increases the demand for the option and its equilibrium price. On the other hand, $\operatorname{CVA}(\phi)$ is a decreasing function of the coverage ratio $\phi$. This implies that the collateral amount increases the demand for the option and its equilibrium volume.

Next, we consider the effect on the price. Differentiating $p^{*}$ in (3.10) with respect to $L$, we have

$$
\frac{\partial p^{*}}{\partial L}=\frac{\gamma_{s}}{B_{T}} \frac{\operatorname{Var}[g(T)]}{\operatorname{CVA}(\phi)}>0 .
$$

Thus, the risk capital $L$ increases the equilibrium price. This effect is due to the change of demand. The increase in the risk capital $L$ increases the demand, while keeping the supply constant.

Based on the analysis in this section, we examine the effect of the counterparty risk constraint and the collateralization on the option contract when the counterparty risk constraint is binding. If the institution provides more than the 
counterparty risk capital to its buyer, the option price and volume increase. These trends arise from the change of demand because the supply is independent of $L$ in our model. On the other hand, for the volume of the contract, if the seller accepts the requirement from the buyer of a higher collateral amount, then the traded amount increases.

In addition to these results, we are interested in the effect of the collateral amount on the equilibrium price when the risk constraint is binding, and the effect of the counterparty risk constraint and the collateralization on the equilibrium price and volume when the risk constraint is not binding. These points are examined in the following numerical example.

\section{Numerical Results}

In this section, we observe the effects of collateralization, the risk capital, and the threshold on the equilibrium of the option contract. In the previous section, we observed the influence of the risk capital on the equilibrium when the risk constraint is binding; that is, the case of $k_{l} \operatorname{CVA}(\phi)=L$. The remaining effects are examined in this section.

\subsection{Model and parameters}

In order to implement our pricing model, we explicitly introduce stochastic price processes for the assets, following Takino (2016b). We consider a filtered probability space $\left(\Omega, \mathcal{F}, P, \mathcal{F}_{t}\right)$ for $0 \leq t \leq T$ and a three-dimensional standard Brownian motion $W=\left(W_{1}, W_{2}, W_{3}\right)$. The filtration $\mathcal{F}_{t}$ is generated by $W_{t}$. Then, we formulate the price processes of assets as follows:

$$
\begin{aligned}
d Y_{u} & =Y_{u}\left(\mu_{Y} d u+\sigma_{Y} d W_{1 u}\right), \quad Y_{0}=Y(>0), \\
d S_{u}^{l} & =S_{u}^{l}\left\{\mu_{l} d u+\sigma_{l}\left(\rho_{l} d W_{1 u}+\sqrt{1-\rho_{l}^{2}} W_{2 u}\right)\right\}, \quad S_{0}^{l}=S_{l}(>0), \\
d S_{u}^{s} & =S_{u}^{s}\left\{\mu_{s} d u+\sigma_{s}\left(\rho_{s} d W_{1 u}+\sqrt{1-\rho_{s}^{2}} W_{3 u}\right)\right\}, \quad S_{0}^{s}=S_{s}(>0),
\end{aligned}
$$

for $0 \leq u \leq T$, where $\mu_{Y}, \sigma_{Y}, \mu_{j}(j=l, s), \sigma_{j}(j=l, s)$, and $\rho_{j}(j=l, s)$ are constant, and $\rho_{j}$ shows the correlation between the asset price for participant $j$ and the underlying asset of the derivatives.

We implement our equilibrium model for the process (4.1) using a Monte-Carlo simulation. The parameters used in the simulation are $\gamma_{l}=0.002, \mu_{l}=0.1, \sigma_{l}=0.2$, $S_{0}^{l}=4000, \quad \pi_{l}=2000, \quad \gamma_{s}=0.001, \quad \mu_{s}=0.4, \quad \sigma_{s}=0.6, \quad S_{0}^{s}=4000, \quad \pi_{s}=2000$, $Y_{0}=100.0, \mu_{Y}=0.1, \sigma_{Y}=0.15, r=0.05$, and $T=1.0$. We consider a European call option with strike price $K$ and its payoff function is

$$
H(T, \omega)=\max \left(Y_{T}(\omega)-K, 0\right) .
$$

We set $K=70.0$. That is, we consider an in-the-money option. The default event of the option seller is supposed to be determined by the terminal asset price held by the seller, as in Henderson \& Liang (2016). That is, the default indicator function is 
defined as

$$
1_{D}(\omega)=1_{S_{T}^{s}(\omega)<L_{s}} .
$$

We set $\eta_{s}=1.0$ and $L_{s}=3000$. The simulation is run 1000000 times.

\subsection{Sensitivity analysis}

Our main purpose is to verify the influence of collateralization on the derivative contract. To this end, we solve the equilibrium formulae of the option contract by changing the coverage ratio $\phi$ for each risk capital $L$ and threshold $M$.

\subsubsection{Effect of risk capital $L$}

We first examine the equilibrium price and volume of the option for each risk capital $L$ without the threshold; that is, we set $M \equiv 0$. In this implementation, we observe the influence of the risk capital on the equilibria only.

Tables 1 and 2, respectively, show the equilibrium volume and price for each $\left(\rho_{l}, \rho_{s}\right)$ and $L$. We examine for $L=1.0,10.0,100.0$. Recall, the risk constraint in our study is

$$
\operatorname{CVA}(\phi) \leq L
$$

Thus, whether the option buyer exposed to a counterparty risk has the restriction of the counterparty risk imposed, depends on $\phi$ and $L$. The increase in $\phi$ reduces the counterparty risk. Hence, if we increase $\phi$, then the constraint of counterparty risk is relaxed, and the amount of counterparty risk is less than $L$. 'Flag' in the tables indicates whether the counterparty risk constraint is binding. The character "B" means that the counterparty risk constraint is binding (i.e. $\operatorname{CVA}(\phi)=L$ ). The character "UB" show that the counterparty risk constraint is not binding (i.e. $\operatorname{CVA}(\phi)<L)$, that is, the situations with no risk constraint. For example, for $L=$ $1.0,10.0$ in the upper table (the case of $\left.\left(\rho_{l}, \rho_{s}\right)=(-0.75,0.75)\right)$ of Table 1, Flag changes from $\mathrm{B}$ to UB when $\phi$ increases. In addition, when $L$ becomes large, Flag changes from $\mathrm{B}$ to $\mathrm{UB}$, since the restriction is relaxed, as mentioned in Sec. 2.4. For example, at $\phi=15 \%$, when $\left(\rho_{l}, \rho_{s}\right)=(-0.75,0.75)$, Flag is UB when $L=100.0$, while Flag is B for $L=1.0,10.0$. In the upper table in Table 1, for instance, the risk constraint is not binding for all $\phi$ when $L=100$, while the risk constraint is binding for $0 \% \leq \phi \leq 15 \%$ at $L=1.0,10.0$.

We first consider the effect of $L$ on the equilibrium volume for the option contract (Table 1). We observe significant differences in volume between the bound risk constraint (Flag B) and when it is not binding (Flag UB). Let's consider the case of $\left(\rho_{l}, \rho_{s}\right)=(-0.75,0.75)$ (the upper table in Table 1) as an example. From the table, the risk constraint is binding for $0 \% \leq \phi \leq 15 \%$ when $L=1.0$, and the volume takes a value from 0.46 to 3.38 . On the one hand, for $20 \% \leq \phi \leq 50 \%$ at $L=1.0$, where the risk constraint is not binding, the volume is between 40.58 and 40.78 . For other $L$, the volumes with no bound risk constraint are larger than the volumes when the risk 
Table 1. Equilibrium volume for each $L$ without the threshold. "Flag" shows whether the counterparty risk constraint is bound or not. Flag is " $\mathrm{B}$ " if the counterparty risk constraint is bound (i.e. $\operatorname{CVA}(\phi)=L$ ), and Flag is "UB" when the risk constraint is not bound (i.e. $\operatorname{CVA}(\phi)<L$ ).

\begin{tabular}{|c|c|c|c|c|c|c|}
\hline \multirow[t]{2}{*}{$\phi$} & \multicolumn{2}{|c|}{$L=1.0$} & \multicolumn{2}{|c|}{$L=10.0$} & \multicolumn{2}{|c|}{$L=100$} \\
\hline & Flag & Volume & Flag & Volume & Flag & Volume \\
\hline \multicolumn{7}{|c|}{$\rho_{l}=-0.75, \rho_{s}=0.75$} \\
\hline $0 \%$ & B & 0.46 & B & 4.65 & UB & 38.61 \\
\hline $5 \%$ & B & 0.65 & $\mathrm{~B}$ & 6.46 & UB & 39.33 \\
\hline $10 \%$ & B & 1.07 & B & 10.73 & UB & 39.93 \\
\hline $15 \%$ & $\mathrm{~B}$ & 3.38 & $\mathrm{~B}$ & 33.77 & UB & 40.25 \\
\hline $20 \%$ & UB & 40.58 & UB & 40.58 & UB & 40.58 \\
\hline $25 \%$ & UB & 40.62 & UB & 40.62 & UB & 40.62 \\
\hline $30 \%$ & UB & 40.65 & UB & 40.65 & UB & 40.65 \\
\hline $35 \%$ & UB & 40.64 & UB & 40.64 & UB & 40.64 \\
\hline $40 \%$ & UB & 40.78 & UB & 40.78 & UB & 40.78 \\
\hline $45 \%$ & UB & 40.73 & UB & 40.73 & UB & 40.73 \\
\hline $50 \%$ & UB & 40.72 & UB & 40.72 & UB & 40.72 \\
\hline \multicolumn{7}{|c|}{$\rho_{l}=-0.5, \rho_{s}=0.5$} \\
\hline $0 \%$ & B & 0.32 & B & 3.21 & UB & 27.29 \\
\hline $5 \%$ & B & 0.41 & $\mathrm{~B}$ & 4.12 & UB & 27.42 \\
\hline $10 \%$ & B & 0.58 & B & 5.83 & UB & 27.54 \\
\hline $15 \%$ & B & 1.02 & $\mathrm{~B}$ & 10.16 & UB & 27.51 \\
\hline $20 \%$ & $\mathrm{~B}$ & 3.82 & UB & 27.57 & UB & 27.57 \\
\hline $25 \%$ & UB & 27.38 & UB & 27.38 & UB & 27.38 \\
\hline $30 \%$ & UB & 27.3 & UB & 27.3 & UB & 27.3 \\
\hline $35 \%$ & UB & 27.18 & UB & 27.18 & UB & 27.18 \\
\hline $40 \%$ & UB & 27.24 & UB & 27.24 & UB & 27.24 \\
\hline $45 \%$ & UB & 27.12 & UB & 27.12 & UB & 27.12 \\
\hline $50 \%$ & UB & 27.09 & UB & 27.09 & UB & 27.09 \\
\hline \multicolumn{7}{|c|}{$\rho_{l}=-0.25, \rho_{s}=0.25$} \\
\hline $0 \%$ & B & 0.25 & B & 2.48 & UB & 16.28 \\
\hline $5 \%$ & B & 0.3 & B & 3.04 & UB & 15.92 \\
\hline $10 \%$ & B & 0.4 & B & 4.01 & UB & 15.61 \\
\hline $15 \%$ & B & 0.59 & B & 5.9 & UB & 15.28 \\
\hline $20 \%$ & B & 1.12 & B & 11.18 & UB & 15.03 \\
\hline $25 \%$ & B & 8.53 & UB & 14.64 & UB & 14.64 \\
\hline $30 \%$ & UB & 14.39 & UB & 14.39 & UB & 14.39 \\
\hline $35 \%$ & UB & 14.13 & UB & 14.13 & UB & 14.13 \\
\hline $40 \%$ & UB & 14.07 & UB & 14.07 & UB & 14.07 \\
\hline $45 \%$ & UB & 13.85 & UB & 13.85 & UB & 13.85 \\
\hline $50 \%$ & UB & 13.78 & UB & 13.78 & UB & 13.78 \\
\hline
\end{tabular}

constraint is binding. These characteristics hold for the case of $\left(\rho_{l}, \rho_{s}\right)=(-0.5,0.5)$. Therefore, for small $\phi$, the increase in $\phi$ increases the traded amount. Recall, the increase in $\phi$ relaxes the risk constraint. Therefore, the volume of the claim increases through relaxing the risk constraint when the coverage ratio $\phi$ increases. It is also interpreted that greater collateralization is preferred to increase liquidity when the 
Table 2. Equilibrium price for each $L$ without the threshold. "Flag" shows whether the counterparty risk constraint is bound or not. Flag is "B" if the counterparty risk constraint is bound (i.e. $\operatorname{CVA}(\phi)=L$ ), and Flag is "UB" when the risk constraint is not bound (i.e. $\operatorname{CVA}(\phi)<L)$.

\begin{tabular}{|c|c|c|c|c|c|c|}
\hline \multirow[t]{2}{*}{$\phi$} & \multicolumn{2}{|c|}{$L=1.0$} & \multicolumn{2}{|c|}{$L=10.0$} & \multicolumn{2}{|c|}{$L=100$} \\
\hline & Flag & Price & Flag & Price & Flag & Price \\
\hline \multicolumn{7}{|c|}{$\rho_{l}=-0.75, \rho_{s}=0.75$} \\
\hline $0 \%$ & B & 13.8 & $\mathrm{~B}$ & 15.06 & UB & 25.27 \\
\hline $5 \%$ & $\mathrm{~B}$ & 14.73 & $\mathrm{~B}$ & 16.41 & UB & 25.9 \\
\hline $10 \%$ & $\mathrm{~B}$ & 15.45 & $\mathrm{~B}$ & 18.16 & UB & 26.33 \\
\hline $15 \%$ & B & 16.62 & $\mathrm{~B}$ & 24.93 & UB & 26.7 \\
\hline $20 \%$ & UB & 26.97 & UB & 26.97 & UB & 26.97 \\
\hline $25 \%$ & UB & 27.13 & UB & 27.13 & UB & 27.13 \\
\hline $30 \%$ & UB & 27.29 & UB & 27.29 & UB & 27.29 \\
\hline $35 \%$ & UB & 27.36 & UB & 27.36 & UB & 27.36 \\
\hline $40 \%$ & UB & 27.29 & UB & 27.29 & UB & 27.29 \\
\hline $45 \%$ & UB & 27.36 & UB & 27.36 & UB & 27.36 \\
\hline $50 \%$ & UB & 27.37 & UB & 27.37 & UB & 27.37 \\
\hline \multicolumn{7}{|c|}{$\rho_{l}=-0.5, \rho_{s}=0.5$} \\
\hline $0 \%$ & B & 20.24 & $\mathrm{~B}$ & 21.1 & UB & 28.23 \\
\hline $5 \%$ & B & 21.24 & $\mathrm{~B}$ & 22.3 & UB & 28.96 \\
\hline $10 \%$ & $\mathrm{~B}$ & 21.97 & $\mathrm{~B}$ & 23.43 & UB & 29.48 \\
\hline $15 \%$ & $\mathrm{~B}$ & 22.68 & $\mathrm{~B}$ & 25.18 & UB & 29.91 \\
\hline $20 \%$ & $\mathrm{~B}$ & 23.9 & UB & 30.29 & UB & 30.29 \\
\hline $25 \%$ & UB & 30.56 & UB & 30.56 & UB & 30.56 \\
\hline $30 \%$ & UB & 30.79 & UB & 30.79 & UB & 30.79 \\
\hline $35 \%$ & UB & 30.97 & UB & 30.97 & UB & 30.97 \\
\hline $40 \%$ & UB & 30.96 & UB & 30.96 & UB & 30.96 \\
\hline $45 \%$ & UB & 31.1 & UB & 31.1 & UB & 31.1 \\
\hline $50 \%$ & UB & 31.13 & UB & 31.13 & UB & 31.13 \\
\hline \multicolumn{7}{|c|}{$\rho_{l}=-0.25, \rho_{s}=0.25$} \\
\hline $0 \%$ & B & 26.5 & $\mathrm{~B}$ & 27.14 & UB & 31.08 \\
\hline $5 \%$ & $\mathrm{~B}$ & 27.55 & $\mathrm{~B}$ & 28.31 & UB & 31.89 \\
\hline $10 \%$ & $\mathrm{~B}$ & 28.35 & $\mathrm{~B}$ & 29.33 & UB & 32.49 \\
\hline $15 \%$ & $\mathrm{~B}$ & 29.04 & $\mathrm{~B}$ & 30.46 & UB & 32.97 \\
\hline $20 \%$ & $\mathrm{~B}$ & 29.76 & $\mathrm{~B}$ & 32.42 & UB & 33.44 \\
\hline $25 \%$ & $\mathrm{~B}$ & 32.18 & UB & 33.79 & UB & 33.79 \\
\hline $30 \%$ & UB & 34.08 & UB & 34.08 & UB & 34.08 \\
\hline $35 \%$ & UB & 34.34 & UB & 34.34 & UB & 34.34 \\
\hline $40 \%$ & UB & 34.41 & UB & 34.41 & UB & 34.41 \\
\hline $45 \%$ & UB & 34.61 & UB & 34.61 & UB & 34.61 \\
\hline $50 \%$ & UB & 34.68 & UB & 34.68 & UB & 34.68 \\
\hline
\end{tabular}

participant is confined by the counterparty risk constraint. Furthermore, from the table, we observe that the volume at $L=100$ dominates the volume at $L=10.0$, and the volume at $L=10.0$ dominates the volume at $L=1.0$. These properties hold for other $\left(\rho_{l}, \rho_{s}\right)$. Thus, the increase in $L$ increases the volume of the claim, as demonstrated by Brunnermeier \& Pedersen (2009) for a general asset. 
Next, we consider the effect of $L$ on the option price (Table 2). The table shows that the equilibrium price monotonically increases with the increase in $\phi$, for all $L$ and $\left(\rho_{l}, \rho_{s}\right)$. From the fact that the increase in $\phi$ relaxes the risk constraint, we conclude that the price of the claim increases through easing the risk constraint when the coverage ratio $\phi$ increases, as in the case of volume. We also have the following result. The prices with lower $L$ are dominated the prices with higher $L$. For example, in the case of $\left(\rho_{l}, \rho_{s}\right)=(-0.75,0.75)$, the prices with $L=1.0$ are lower than the prices with $L=10.0$, and the prices with $L=10.0$ are lower than the prices with $L=100$. This feature holds for other $\left(\rho_{l}, \rho_{s}\right)$. Hence, the increase in risk capital $L$ increases the option price.

From the above results, the increase in risk capital $L$ for the counterparty risk boosts both the price and the volume of the claim. This risk capital means the upper limit enables the buyer of the claim to take a counterparty risk up to this amount. That is, the buyer is able to have the derivatives contract up to risk capital $L$. Therefore, the increase in risk capital $L$ causes the increase in demand and increases the price and the volume. In fact, when the counterparty risk constraint is binding, the demand $k$ for the claim is given by

$$
k=\frac{L}{\operatorname{CVA}(\phi)} .
$$

Hence, the increase in $L$ increases $k$, that is, the demand for the claim.

\subsubsection{Effect of threshold $M$}

We consider the effect of the threshold $M$ on the derivative contract. In this simulation, we fix the allocated risk capital $L=10.0$. Tables 3 and 4 respectively show equilibrium volume and price when threshold $M$ is applied.

As shown in Sec. 2.4, the increase in the threshold $M$ increases the counterparty risk measured by the CVA. Thus, the increase in threshold $M$ makes the counterparty risk constraint binding (denoted by Flag B in Tables 3 and 4 ). In fact, for the case of $\left(\rho_{l}, \rho_{s}\right)=(-0.75,0.75)$, the constraint is bound for $0 \% \leq \phi \leq 15 \%$ at $M=0.0,0 \% \leq \phi \leq 35 \%$ at $M=20.0$, and all $\phi$ at $M=40.0$. That is, the range of $\phi$ where the risk constraint is binding expands with the increase in $M$. For other cases of $\left(\rho_{l}, \rho_{s}\right)$, the results are the same as the case of $\left(\rho_{l}, \rho_{s}\right)=(-0.75,0.75)$. We also have noncollateralized transactions for large $M$ (not shown). At $M=40$, when $\left(\rho_{l}, \rho_{s}\right)=(-0.75,0.75)$, we have

$$
V_{0}=0 \quad \text { or } \tilde{V}_{0}<M
$$

for all $\phi$. Then, the equilibrium formulae are independent of the coverage ratio $\phi$ and the amount of collateral. In the columns of $M=40$ at $\left(\rho_{l}, \rho_{s}\right)=(-0.75,0.75)$ in Tables 3 and 4, the volume and price do not depend on $\phi$.

We first consider the effect of $M$ on the equilibrium volume of the option contract. Table 3 demonstrates a quantitative characteristic of volume when the risk 


\section{K. Takino}

Table 3. Equilibrium volume for each $M$ at $L=10.0$. "Flag" shows whether the counterparty risk constraint is bound or not. Flag is "B" if the counterparty risk constraint is bound (i.e. $\operatorname{CVA}(\phi)=L$ ), and Flag is "UB" when the risk constraint is not bound (i.e. $\operatorname{CVA}(\phi)<L)$.

\begin{tabular}{|c|c|c|c|c|c|c|}
\hline \multirow[t]{3}{*}{$\phi$} & \multicolumn{6}{|c|}{$\rho_{l}=-0.75, \rho_{s}=0.75$} \\
\hline & \multicolumn{2}{|c|}{$M=0.0$} & \multicolumn{2}{|c|}{$M=20.0$} & \multicolumn{2}{|c|}{$M=40.0$} \\
\hline & Flag & Volume & Flag & Volume & Flag & Volume \\
\hline $0 \%$ & B & 4.65 & B & 4.65 & B & 4.65 \\
\hline $5 \%$ & B & 6.46 & B & 5.19 & B & 4.61 \\
\hline $10 \%$ & B & 10.73 & B & 5.91 & B & 4.59 \\
\hline $15 \%$ & B & 33.77 & B & 6.98 & B & 4.63 \\
\hline $20 \%$ & UB & 40.58 & B & 8.39 & B & 4.62 \\
\hline $25 \%$ & UB & 40.62 & B & 10.49 & B & 4.6 \\
\hline $30 \%$ & UB & 40.65 & B & 13.93 & B & 4.6 \\
\hline $35 \%$ & UB & 40.64 & $\mathrm{~B}$ & 21.33 & B & 4.61 \\
\hline $40 \%$ & UB & 40.78 & UB & 40.4 & B & 4.6 \\
\hline $45 \%$ & UB & 40.73 & UB & 40.46 & B & 4.63 \\
\hline \multirow[t]{4}{*}{$50 \%$} & UB & 40.72 & UB & 40.53 & B & 4.61 \\
\hline & \multicolumn{6}{|c|}{$\rho_{l}=-0.5, \rho_{s}=0.5$} \\
\hline & \multicolumn{2}{|c|}{$M=0.0$} & \multicolumn{2}{|c|}{$M=15.0$} & \multicolumn{2}{|c|}{$M=30.0$} \\
\hline & Flag & Volume & Flag & Volume & Flag & Volume \\
\hline $0 \%$ & B & 3.21 & B & 3.21 & B & 3.21 \\
\hline $5 \%$ & B & 4.12 & B & 3.66 & B & 3.29 \\
\hline $10 \%$ & B & 5.83 & B & 4.28 & B & 3.39 \\
\hline $15 \%$ & $\mathrm{~B}$ & 10.16 & B & 5.24 & B & 3.53 \\
\hline $20 \%$ & UB & 27.57 & B & 6.7 & B & 3.67 \\
\hline $25 \%$ & UB & 27.38 & B & 9.1 & B & 3.78 \\
\hline $30 \%$ & UB & 27.3 & $\mathrm{~B}$ & 14.34 & B & 3.93 \\
\hline $35 \%$ & UB & 27.18 & UB & 27.43 & B & 4.11 \\
\hline $40 \%$ & UB & 27.24 & UB & 27.52 & B & 4.26 \\
\hline $45 \%$ & UB & 27.12 & UB & 27.41 & B & 4.48 \\
\hline \multirow[t]{4}{*}{$50 \%$} & UB & 27.09 & UB & 27.38 & B & 4.67 \\
\hline & \multicolumn{6}{|c|}{$\rho_{l}=-0.25, \rho_{s}=0.25$} \\
\hline & \multicolumn{2}{|c|}{$M=0.0$} & \multicolumn{2}{|c|}{$M=5.0$} & \multicolumn{2}{|c|}{$M=10.0$} \\
\hline & Flag & Volume & Flag & Volume & Flag & Volume \\
\hline $0 \%$ & B & 2.48 & B & 2.48 & B & 2.48 \\
\hline $5 \%$ & B & 3.04 & B & 2.94 & B & 2.85 \\
\hline $10 \%$ & B & 4.01 & B & 3.69 & B & 3.42 \\
\hline $15 \%$ & B & 5.9 & B & 4.96 & B & 4.28 \\
\hline $20 \%$ & $\mathrm{~B}$ & 11.18 & $\mathrm{~B}$ & 7.56 & B & 5.71 \\
\hline $25 \%$ & UB & 14.64 & UB & 14.84 & B & 8.37 \\
\hline $30 \%$ & UB & 14.39 & UB & 14.6 & UB & 14.83 \\
\hline $35 \%$ & UB & 14.13 & UB & 14.34 & UB & 14.58 \\
\hline $40 \%$ & UB & 14.07 & UB & 14.27 & UB & 14.51 \\
\hline $45 \%$ & UB & 13.85 & UB & 14.04 & UB & 14.27 \\
\hline $50 \%$ & UB & 13.78 & UB & 13.94 & UB & 14.17 \\
\hline
\end{tabular}


Table 4. Equilibrium price for each $M$ at $L=10.0$. "Flag" shows whether the counterparty risk constraint is bound or not. Flag is " $\mathrm{B}$ " if the counterparty risk constraint is bound (i.e. $\operatorname{CVA}(\phi)=L$ ), and Flag is "UB" when the risk constraint is not bound (i.e. $\operatorname{CVA}(\phi)<L)$.

\begin{tabular}{|c|c|c|c|c|c|c|}
\hline \multirow[t]{3}{*}{$\phi$} & \multicolumn{6}{|c|}{$\rho_{l}=-0.75, \rho_{s}=0.75$} \\
\hline & \multicolumn{2}{|c|}{$M=0.0$} & \multicolumn{2}{|c|}{$M=20.0$} & \multicolumn{2}{|c|}{$M=40.0$} \\
\hline & Flag & Price & Flag & Price & Flag & Price \\
\hline $0 \%$ & B & 15.06 & B & 15.06 & B & 15.06 \\
\hline $5 \%$ & B & 16.41 & B & 15.58 & B & 15.08 \\
\hline $10 \%$ & B & 18.16 & B & 16.01 & B & 14.98 \\
\hline $15 \%$ & B & 24.93 & B & 16.61 & B & 15.01 \\
\hline $20 \%$ & UB & 26.97 & B & 17.25 & B & 15 \\
\hline $25 \%$ & UB & 27.13 & B & 18.06 & B & 14.98 \\
\hline $30 \%$ & UB & 27.29 & B & 19.29 & B & 15.08 \\
\hline $35 \%$ & UB & 27.36 & B & 21.49 & B & 15.06 \\
\hline $40 \%$ & UB & 27.29 & UB & 26.68 & B & 14.92 \\
\hline $45 \%$ & UB & 27.36 & UB & 26.85 & B & 14.99 \\
\hline \multirow[t]{4}{*}{$50 \%$} & UB & 27.37 & UB & 26.93 & B & 14.97 \\
\hline & \multicolumn{6}{|c|}{$\rho_{l}=-0.5, \rho_{s}=0.5$} \\
\hline & \multicolumn{2}{|c|}{$M=0.0$} & \multicolumn{2}{|c|}{$M=15.0$} & \multicolumn{2}{|c|}{$M=30.0$} \\
\hline & Flag & Price & Flag & Price & Flag & Price \\
\hline $0 \%$ & B & 21.1 & B & 21.1 & B & 21.1 \\
\hline $5 \%$ & B & 22.3 & B & 21.81 & B & 21.31 \\
\hline $10 \%$ & B & 23.43 & B & 22.38 & B & 21.4 \\
\hline $15 \%$ & $\mathrm{~B}$ & 25.18 & B & 23.05 & B & 21.55 \\
\hline $20 \%$ & UB & 30.29 & B & 23.82 & B & 21.71 \\
\hline $25 \%$ & UB & 30.56 & B & 24.82 & B & 21.87 \\
\hline $30 \%$ & UB & 30.79 & B & 26.56 & B & 22.08 \\
\hline $35 \%$ & UB & 30.97 & UB & 30.33 & B & 22.26 \\
\hline $40 \%$ & UB & 30.96 & UB & 30.39 & B & 22.27 \\
\hline $45 \%$ & UB & 31.1 & UB & 30.6 & B & 22.53 \\
\hline \multirow[t]{4}{*}{$50 \%$} & UB & 31.13 & UB & 30.7 & B & 22.65 \\
\hline & \multicolumn{6}{|c|}{$\rho_{l}=-0.25, \rho_{s}=0.25$} \\
\hline & \multicolumn{2}{|c|}{$M=0.0$} & \multicolumn{2}{|c|}{$M=5.0$} & \multicolumn{2}{|c|}{$M=10.0$} \\
\hline & Flag & Price & Flag & Price & Flag & Price \\
\hline $0 \%$ & B & 27.14 & B & 27.14 & B & 27.14 \\
\hline $5 \%$ & B & 28.31 & B & 28.16 & B & 28.02 \\
\hline $10 \%$ & B & 29.33 & B & 29.04 & B & 28.75 \\
\hline $15 \%$ & B & 30.46 & B & 29.95 & B & 29.49 \\
\hline $20 \%$ & $\mathrm{~B}$ & 32.42 & B & 31.18 & B & 30.37 \\
\hline $25 \%$ & UB & 33.79 & UB & 33.56 & B & 31.52 \\
\hline $30 \%$ & UB & 34.08 & UB & 33.86 & UB & 33.59 \\
\hline $35 \%$ & UB & 34.34 & UB & 34.13 & UB & 33.87 \\
\hline $40 \%$ & UB & 34.41 & UB & 34.22 & UB & 33.98 \\
\hline $45 \%$ & UB & 34.61 & UB & 34.45 & UB & 34.22 \\
\hline $50 \%$ & UB & 34.68 & UB & 34.54 & UB & 34.34 \\
\hline
\end{tabular}




\section{K. Takino}

constraint is and is not binding. As observed in the previous section, the volumes under the bound risk constraint are lower than those with the unbound constraint. For example, for $20 \% \leq \phi \leq 35 \%$ in the case of $\left(\rho_{l}, \rho_{s}\right)=(-0.75,0.75)$, the volumes under $M=0.0$ are larger than those under $M=20.0$. For $40 \% \leq \phi \leq 50 \%$, the volumes under $M=0.0$ and $M=20.0$ are larger than those under $M=40.0$. Since the increase in the threshold makes the risk constraint binding because of deteriorating counterparty risk, the increase in the threshold decreases the volume of the option. Furthermore, for $0 \% \leq \phi \leq 15 \%$ in the case of $\left(\rho_{l}, \rho_{s}\right)=(-0.75,0.75)$, the volumes under $M=0.0$ dominate those under $M=20.0$. For $0 \% \leq \phi \leq 35 \%$ in the case of $\left(\rho_{l}, \rho_{s}\right)=(-0.75,0.75)$, the volumes under $M=20.0$ dominate those under $M=40.0$. That is, when the risk constraint is binding, the volume with a higher threshold $M$ is larger than the volume under a lower threshold $M$. These properties stand for other sets of $\left(\rho_{l}, \rho_{s}\right)$. Therefore, we can conclude that the increase in the threshold decreases the volume of the claim.

Next, we consider the influence on the equilibrium price (Table 4). We consider the case of $\left(\rho_{l}, \rho_{s}\right)=(-0.75,0.75)$ as an example. The prices for $M=0.0$ are larger than the prices for $M=20.0$, for all $\phi$, and the prices for $M=20.0$ are larger than the prices for $M=40.0$, for all $\phi$. These characteristics are observed for other cases of $\left(\rho_{l}, \rho_{s}\right)$. Therefore, the increase in the threshold decreases the price of the claims.

From the above results, the increase in the threshold $M$ decreases both the price and the volume of the claim. The threshold in our study means the maximum amount of the counterparty risk such that the participant with a positive exposure (i.e. the buyer) does not require collateral, as long as the counterparty risk is less than the threshold. Thus, the buyer might run out her/his capital up to the threshold if the counterparty defaults. Therefore, because lifting the threshold forces the buyer to lose more wealth more as a result of the counterparty's default, the increase in the threshold makes the buyer unwilling to enter into a contract; that is, it decreases the demand for the claim. Thus, the increases in the threshold decreases the price and volume.

\subsection{Proxy for risk aversion}

The previous sections show that an increase in $L$ increases both the volume and the price of the claim, and an increase in $M$ decreases both the volume and the price of the claim. That is, an increase in risk capital or a decrease in the threshold increases the market size $^{c}$ of the option contract under our counterparty risk model. Conversely, we can decrease risk capital or increase the threshold if we want to prevent bulging in the market. From this result, just as risk capital endogenously leads to a

\footnotetext{
${ }^{\mathrm{c}}$ In this study, we simply define the market size as the product of price and volume of the option, while, in practice, the size is referred as either the notional amounts or the gross market value (BIS 2017). Our definition of market size corresponds to the gross market value since our derivative contract is an option that the buyer has a positive exposure in the contract.
} 
spillover effect (Shin 2010), the amount of risk capital affects the behavior of investors in the derivatives market too.

The risk-averse coefficient shows the attitude to risk and influences the behavior of investors in financial markets. In fact, our equilibrium formulae include risk aversion $\gamma_{j}(j=l, s)$ of the market participants. Therefore, we investigate how the risk aversion of participants influences the equilibria. The goal of this examination is to verify whether the allocation of risk capital and the level of the threshold are proxies of risk aversion.

\subsubsection{Equilibrium formulae without collateralization}

We investigate the effect of risk aversion on the derivative transaction. To this end, we reconstruct the equilibrium formulae of the claim, excluding the influence of collateralization; that is, we derive the equilibrium formulae under counterparty risk without the collateral agreement.

First, the terminal wealth of participant $j(j=l, s)$ without collateralization is

$$
W_{T}^{j}(\omega)=w_{j} B_{T}+\frac{\pi_{j}}{S_{0}^{j}} S_{T}^{j}(\omega)+\delta_{j} k_{j} \hat{H}(T, \omega),
$$

where

$$
w_{j}=x_{j}-\pi_{j}-\delta_{j} k_{j} p
$$

$\delta_{l}=1$, and $\delta_{s}=-1$.

Next, we derive the demand and supply functions for the claim, and the market equilibrium. As in Sec. 3, we give the demand and supply functions by solving utility-maximization problems with a mean-variance criterion and the equilibrium price and volume formulae for the claim. Consequently, we have the equilibrium price,

$$
p^{*}=\frac{1}{B_{T}} E[\hat{H}(T)]-\frac{1}{B_{T}} \gamma \operatorname{Cov}\left[R_{T}, \hat{H}(T)\right],
$$

and the equilibrium volume,

$$
k^{*}=\frac{\gamma_{s} \frac{\pi_{s}}{S_{0}^{s}} \operatorname{Cov}\left[S_{T}^{s}, \hat{H}(T)\right]-\gamma_{l} \frac{\pi_{l}}{S_{0}^{l}} \operatorname{Cov}\left[S_{T}^{l}, \hat{H}(T)\right]}{\left(\gamma_{l}+\gamma_{s}\right) \operatorname{Var}[\hat{H}(T)]} .
$$

\subsubsection{Numerical result}

Table 5 shows the result of the Monte-Carlo simulation for the equilibrium price (4.2) and volume (4.3) with stochastic model (4.1). We only examine changing the risk aversion $\gamma_{l}$ of the buyer because only the buyer of the option has counterparty risk in our model.

From the table, we observe that the increase in $\gamma_{l}$ decreases both the price and volume of the claim for each $\left(\rho_{l}, \rho_{s}\right)$. For example, in the case of 
Table 5. Equilibrium price and volume without collateral agreement.

\begin{tabular}{|c|c|c|c|c|c|c|}
\hline \multirow[t]{2}{*}{$\gamma_{l}$} & \multicolumn{2}{|c|}{$\begin{array}{c}\rho_{l}=-0.75 \\
\rho_{s}=0.75\end{array}$} & \multicolumn{2}{|c|}{$\begin{array}{c}\rho_{l}=-0.5 \\
\rho_{s}=0.5\end{array}$} & \multicolumn{2}{|c|}{$\begin{array}{c}\rho_{l}=-0.25 \\
\rho_{s}=0.25\end{array}$} \\
\hline & Price & Volume & Price & Volume & Price & Volume \\
\hline 0.001 & 28.33 & 48.79 & 30.47 & 34.89 & 32.53 & 21.40 \\
\hline 0.002 & 25.27 & 38.58 & 28.25 & 27.23 & 31.11 & 16.21 \\
\hline 0.003 & 23.73 & 33.49 & 27.15 & 23.44 & 30.42 & 13.66 \\
\hline 0.004 & 22.81 & 30.44 & 26.42 & 21.19 & 29.91 & 12.18 \\
\hline 0.005 & 22.19 & 28.40 & 25.97 & 19.67 & 29.61 & 11.16 \\
\hline 0.006 & 21.76 & 26.96 & 25.65 & 18.62 & 29.40 & 10.48 \\
\hline 0.007 & 21.39 & 25.86 & 25.41 & 17.79 & 29.27 & 9.91 \\
\hline 0.008 & 21.21 & 25.00 & 25.29 & 17.15 & 29.20 & 9.47 \\
\hline 0.009 & 20.90 & 24.34 & 25.03 & 16.66 & 29.01 & 9.16 \\
\hline 0.010 & 20.75 & 23.76 & 24.91 & 16.22 & 28.92 & 8.85 \\
\hline
\end{tabular}

$\left(\rho_{l}, \rho_{s}\right)=(-0.75,0.75)$, the price monotonically decreases from 28.33 to 20.75 , and the volume monotonically decreases from 48.79 to 23.76 when $\gamma_{l}$ increases from 0.001 to 0.010. This characteristic holds for other sets of $\left(\rho_{l}, \rho_{s}\right)$. Thus, when the buyer becomes more risk averse in our option market, the market reduces in size. Combining the above results, in order to avoid a boom or bubble, limiting risk capital or increasing the threshold have the equivalent effect of investors becoming more risk averse. Who, however, can control an investor's attitude to risk? It might be difficult to do so in practice. Our study implies that risk capital and the threshold can be proxies for the risk aversion of investors.

\section{Summary}

We consider an equilibrium model for an OTC option market with counterparty risk, collateralization, the counterparty risk constraint, and the threshold. Our study verified the influences of collateralization, the risk constraint, and the threshold on the derivative contract.

We obtain two main results. The first is that an increase in collateral increases the price and volume of the option for relatively small collateral amounts. The second is that an increase in risk capital or a decrease in the threshold increases the price and volume of an option. The key to these results is whether the risk constraint is binding. In fact, our numerical result shows that the price and volume when the risk constraint is not binding are larger than when it is binding. An increase in collateral reduces the counterparty risk, which relaxes the risk constraint. The reason why the increase in risk capital eases the risk constraint is straightforward. However, the influence of the threshold might seem unintuitive. An increase in the threshold provides more opportunity to sell the option because the seller is no longer required to provide collateral. However, this results in deteriorating counterparty risk owed by the option buyer. Conversely, a decrease in the threshold increases the required 
collateral amount and relaxes the risk constraint. The first statement has been shown in a previous study. This study, however, explicitly demonstrates the same finding from the viewpoint of the risk constraint. The second assertion implies that if we want to increase the market size of the OTC derivative market, we should have more risk capital for counterparty risk or we should reduce the threshold.

We also analyzed the influence of risk aversion on the option contract without collateralization. Here, we only examined the risk aversion of the buyer because she/he has a positive exposure in the derivative contract. From our numerical results, when the buyer of the option becomes more risk averse, the size of the option market decreases. This coincides with the effect of the decrease in risk capital or the increase in the threshold. That is, we provide evidence that the allocation of risk capital or an adjustment of the threshold might be proxies for controlling the risk aversion of market participants who face counterparty risk.

Finally, we have considered the case of a single contract between participants in this study. On the other hand, in practice, different contracts are generated between the same participants. That is, the considered financial product is extended from a single derivatives contract to a portfolio of various contracts. Our study can accommodate a portfolio of contracts because our results are obtained using a Monte-Carlo simulation. However, when we consider the particular case of a portfolio of different contracts with different underlying assets, the Monte-Carlo simulation takes longer to obtain results because we need to simulate price processes of the underlying assets. Furthermore, if different derivatives are made between the same participants, we are able to introduce a netting clause. That is, the amount of counterparty risk is calculated for the portfolio of contracts rather than for the sum of the counterparty risk for each product. Hence, the netting is expected to reduce the counterparty risk. This leads to the participant being released from the counterparty risk constraint. The model in which the participants have different contracts will be considered in future research.

\section{Acknowledgment}

This work was supported by JSPS KAKENHI Grant Number 17K18219. The author also appreciates Dr. Soichiro Moridaira for a helpful comment.

\section{References}

V. Acharya \& A. Bisin (2014) Counterparty risk externality: Centralized versus over-thecounter markets, Journal of Economic Theory 149, 153-182.

H. Bessembinder \& M. L. Lemmon (2002) Equilibrium pricing and optimal hedging in electricity forward markets, The Journal of Finance 57, 1347-1382.

Basel Committee on Banking Supervision (BCBS) (2010) Basel III: A Global Regulatory Framework for more Resilient Banks and Banking Systems, Bank for International Settlements. Available at https://www.bis.org/publ/bcbs189_dec2010.htm

BIS (2017) BIS Statistical Bulletin. September 2017, Bank for International Settlements. Available at www.bis.org/statistics/bulletin1709.pdf 


\section{K. Takino}

D. Brigo \& M. Masetti (2005) Risk neutral pricing of counterparty risk. In: Counterparty Credit Risk Modeling: Risk Management, Pricing and Regulation. London: Risk Books.

M. K. Brunnermeier \& L. H. Pedersen (2009) Market liquidity and funding liquidity, The Review of Financial Studies 22, 2201-2238.

H. Bühlmann (1980) An economic premium principle, Austin Bulletin 11, 52-60.

A. Buss, B. Dumas, R. Uppal \& G. Vilkov (2016) The intended and unintended consequences of financial-market regulations: A general-equilibrium analysis, Journal of Monetary Economics 81, 25-43.

J. Danielsson, H. S. Shin \& J. P. Zigrand (2004) The impact of risk regulation on price dynamics, Journal of Banking and Finance 28, 1069-1087.

J. Danielsson, H. S. Shin \& J. P. Zigrand (2010) Risk appetite and endogenous risk, FMG Discussion Papers, dp647.

M. Fujii \& A. Takahashi (2013) Derivative pricing under asymmetric and imperfect collateralization and CVA, Quantitative Finance 13, 749-768.

J. Geanakoplos (1997) "Promises, Promises", The Economy as an Evolving Complex System II, pp. 285-320.

J. Gregory (2010) Counterparty Credit Risk: The New Challenge for Global Financial Markets. New York: Wiley.

V. Henderson \& G. Liang (2016) A multidimensional exponential utility indifference pricing model with application to counterparty risk, SIAM Journal on Control and Optimization 54, 690-717.

M. Illing \& M. Aaron (2005) A brief survey of risk-appetite indexes, Bank of Canada Financial System Review - June 2005, 37-43.

H. Iwaki, M. Kijima \& Y. Morimoto (2001) An economic premium principle in a multiperiod economy, Insurance: Mathematics and Economics 28, 325-339.

H. Iwaki (2002) An economic premium principle in a continuous-time economy, Journal of the Operations Research Society of Japan 45, 346-361.

M. Johannes \& S. Sundaresan (2003) The impact of collateralization on swap rates, Journal of Finance 62, 383-410.

M. Kijima, A. Maeda \& K. Nishide (2010) Equilibrium pricing of contingent claims in tradable permit markets, Journal of Futures Markets 30, 559-589.

H. S. Shin (2010) Risk and Liquidity (Clarendon Lectures in Finance). New York: Oxford University Press.

K. Takino (2016a) An equilibrium pricing for OTC derivatives with collateralization. Application of economic premium principle, The Soka Economic Studies 45, 61-72.

K. Takino (2016b) An equilibrium model for the OTC derivatives market with a collateral agreement, Journal of Commodity Markets 4, 41-55.

Q. Zhang \& Y. Gao (2017) Portfolio selection based on a benchmark process with dynamic value-at-risk constraints, Journal of Computational and Applied Mathematics $\mathbf{3 1 3}$, 440-447. 